\title{
PRIMITIVE RECURSIVE FUNCTIONS
}

\author{
RAPHAEL M. ROBINSON
}

1. Definition of recursive functions. In this paper, we shall consider certain reductions in the recursion scheme for defining primitive recursive functions. Hereafter, we shall refer to such functions simply as recursive functions. ${ }^{1}$ In $\$ 1$, we define what is meant by a recursive function, and also define some recursive functions which will be used. The statement of the principal results of the paper will be found in $\$ 2$.

By a number, we shall mean one of the natural numbers 0,1 , $2,3, \cdots$ We shall consider functions of any number of variables, each variable ranging over all numbers, and the values of the function being numbers. Small letters will denote variables assuming numerical values, and capital letters will denote functions. In the case of a function of one variable, we shall usually write $F x$ instead of $F(x)$.

A function will be called recursive if it can be obtained from certain initial functions by repeated substitution and recursion.

The initial functions are the following:

The identity functions; that is, for every $n$ and $k$ with $1 \leqq k \leqq n$, the function $I_{n k}$ defined by

$$
I_{n k}\left(x_{1}, \cdots, x_{n}\right)=x_{k} .
$$

The zero functions; that is, for every $n \geqq 0$, the function $O_{n}$ defined by

$$
O_{n}\left(x_{1}, \cdots, x_{n}\right)=0 .
$$

The successor function; that is, the function $S$ of one variable, such that $S x$ is the next number after $x$.

The substitution rule is the following: If $A_{1}, \cdots, A_{m}$ are known functions of $n$ variables, and $B$ is a known function of $m$ variables,

Presented to the Society, August 22, 1946; received by the editors March 5, 1947.

1 Various types of recursive functions play a fundamental role in mathematical logic. Primitive recursive functions were used by K. Gödel, Über formal unentscheidbare Sätze der Principia Mathematica und verwandter Systeme I, Monatshefte für Mathematik und Physik vol. 38 (1931) pp. 173-198; for the definition, see pp. 179180. For a discussion of general recursive functions (which may be considered to be the computable functions), see S. C. Kleene, General recursive functions of natural numbers, Math. Ann. vol. 112 (1936) pp. 727-742. For some other types of recursion, see the paper by $\mathrm{R}$. Péter cited in footnote 2 . In the present paper, we consider only the simplest type of recursive function, the primitive recursive function, and no knowledge of other papers is assumed. 
then a function $F$ of $n$ variables may be defined by

$$
F\left(x_{1}, \cdots, x_{n}\right)=B\left(A_{1}\left(x_{1}, \cdots, x_{n}\right), \cdots, A_{m}\left(x_{1}, \cdots, x_{n}\right)\right) .
$$

It is not excluded that $m$ or $n$ should be zero.

In particular, we can obtain by substitution the functions

$$
\mathrm{SO}_{n}\left(x_{1}, \cdots, x_{n}\right), \operatorname{SSO}_{n}\left(x_{1}, \cdots, x_{n}\right), \cdots,
$$

that is, all constant functions. Also, if we put $O_{m}$ for $B$, and any functions of $n$ variables for $A_{1}, \cdots, A_{m}$, then $F$ is the function $O_{n}$. Thus it was not necessary to assume all the zero functions as initial functions; we could in fact have taken just $O_{0}$.

Furthermore, using the identity functions, any desired permutation of variables, or introduction of extra variables, may be accomplished. For example, given a function $B$ of two variables, suppose it is required to define a function $F$ of three variables, such that

$$
F(x, y, z)=B(z, x) .
$$

This is seen to be included in our substitution rule when written in the form

$$
F(x, y, z)=B\left(I_{33}(x, y, z), I_{31}(x, y, z)\right) .
$$

Finally, we must tell what is meant by recursion. If $A$ is a known function of $n$ variables, and $B$ a known function of $n+2$ variables, then a function $F$ of $n+1$ variables may be defined by the recursion scheme

$$
\begin{aligned}
F\left(u_{1}, \cdots, u_{n}, 0\right) & =A\left(u_{1}, \cdots, u_{n}\right), \\
F\left(u_{1}, \cdots, u_{n}, S x\right) & =B\left(u_{1}, \cdots, u_{n}, x, F\left(u_{1}, \cdots, u_{n}, x\right)\right) .
\end{aligned}
$$

The recursion is said to be with respect to $x$; the other variables $u_{1}, \cdots, u_{n}$ are called parameters. In case $n=0$, we may replace $A\left(u_{1}, \cdots, u_{n}\right)$ by a number $a$. This completes the definition of what is meant by a recursive function.

Familiar examples of recursive definitions with one parameter are those of $u+x, u x$, and $u^{x}$, which must be made in this order. The definitions are:

$u+x: u+0=u, u+S x=S(u+x)$; that is, $A u=u, B(u, x, y)=S y$.

$u x: \quad u \cdot 0=0, \quad u \cdot S x=u x+u$; that is, $A u=0, B(u, x, y)=y+u$.

$u^{x}: \quad u^{0}=1, \quad u^{S x}=w^{x} \cdot u ; \quad$ that is, $A u=1, B(u, x, y)=y u$.

We shall make much use of the function $0^{x}$, and also of $\operatorname{sgn} x=0^{0^{x}}$.

We shall also use predecessor and difference, defined by 


$$
\begin{aligned}
& \text { Px: } \quad P 0=0, \quad P S x=x ; \\
& u-x: \quad u \div 0=u, \quad u-S x=P(u-x) .
\end{aligned}
$$

Notice that $u-x=0$ if $u<x$. We also use the absolute difference

$$
|u-x|=(u-x)+(x-u) \text {. }
$$

The notation $u-x$, without dot or vertical bars, will always be used in an ambiguous sense, to stand for any recursive function $F(u, x)$ which is equal to $u-x$ for $u \geqq x$, regardless of its value when $u<x$. Any such function will be called a difference function. In particular, $u-x$ and $|u-x|$ are difference functions.

The functions $D x$ and $T x$, giving the remainders when $x$ is divided by 2 or by 3 , are defined by

$$
\begin{array}{lll}
D x: & D 0=0, & D S x=0^{D x} ; \\
T x: & T 0=0, & T S x=0^{T x}+2 \cdot 0^{|T x-1|} .
\end{array}
$$

We may also describe $D$ as the characteristic function of odd numbers. Two further recursive definitions are

$$
\begin{array}{lll}
F x=[x / 2]: & F 0=0, & F S x=F x+D x \\
F x=\left[x^{1 / 2}\right]: & F 0=0, & F S x=F x+0^{(S F x)^{2}-S x} .
\end{array}
$$

In the last definition, $F S x$ is obtained from $F x$ by adding 1 when the next square after $x$ is the next number after $x$, but adding 0 when the next square is larger than the next number. We may now obtain by substitution the excess over a square, and the characteristic function of squares:

$$
E x=x-\left[x^{1 / 2}\right]^{2}, \quad Q x=0^{E x} .
$$

All of the symbols defined in this section will be used with the same meaning throughout the paper. The letters $J, K, L$ will have a special meaning defined in $\$ \S 3$ and 4 . On the other hand, the symbols $A, B$, $F$ will be reserved for general functions, and may have a different meaning each time they are used.

2. Statement of principal results. We shall consider certain more special recursion schemes than the one which occurs in the definition of recursive functions. One restriction is to limit the number of parameters. Another restriction is to suppose that $B$ does not depend on all of its variables. If $B\left(u_{1}, \cdots, u_{n}, x, y\right)$ does not depend on the parameters $u_{1}, u_{2} \cdots, u_{n}$, we shall call the recursion scheme iteration. (If $n=0$, this is no restriction.) If $B\left(u_{1}, \cdots, u_{n}, x, y\right)$ does not depend on $x$, we shall speak of pure recursion, and if it depends only on 
$y$, of pure iteration. Thus with one parameter, we have the four recursion schemes:

$\begin{array}{lll}\text { Recursion: } & F(u, 0)=A u, & F(u, S x)=B(u, x, F(u, x)) . \\ \text { Pure Recursion: } & F(u, 0)=A u, & F(u, S x)=B(u, F(u, x)) . \\ \text { Iteration: } & F(u, 0)=A u, & F(u, S x)=B(x, F(u, x)) . \\ \text { Pure Iteration: } & F(u, 0)=A u, & F(u, S x)=B F(u, x) .\end{array}$

It should be noticed, here and below, that the more special schemes are always actually included in the more general ones, since (by using the identity functions) a function of certain variables may always be considered as a function of more variables.

The last two schemes are no less general, if taken in the form:

Iteration:

$$
\begin{array}{lll}
\text { Iteration: } & F(u, 0)=u, & F(u, S x)=B(x, F(u, x)) . \\
\text { Pure Iteration: } & F(u, 0)=u, & F(u, S x)=B F(u, x) .
\end{array}
$$

For it is easily seen that if $F(u, x)$ is defined in this way, then the previously defined function is simply $F(A u, x)$.

The function $F(u, x)$ obtained by the last scheme (pure iteration) is simply the result of applying the function $B x$ times to $u$. Hence we write

$$
F(u, x)=B^{x} u
$$

For example, $u+x=S^{x} u$.

With no parameter, we have but two recursion schemes, since there is no distinction between recursion and iteration. These are:

Recursion: $\quad F 0=a, \quad F S x=B(x, F x)$.

Pure Recursion: $\quad F 0=a, \quad F S x=B F x$.

Since $n=0$, the function $A\left(u_{1}, \cdots, u_{n}\right)$ is replaced by the number $a$. The pure recursion scheme defines the function $F x=B^{x} a$. Unlike $B^{x} u$ above, this is a function of the one variable $x$, since $a$ is a given number.

We also consider the somewhat more special schemes where only the value 0 is allowed for $a$ :

Recursion: $\quad F 0=0, \quad F S x=B(x, F x)$.

Pure Recursion: $\quad F 0=0, \quad F S x=B F x$.

Even the last and most special recursion scheme, $F x=B^{x} 0$, will be seen to be adequate to define all recursive functions, if two functions are adjoined to the initial functions. 
For each degree of specialization of the recursion scheme, we shall ask what recursive functions need to be adjoined to the initial functions, in order that all recursive functions can be defined. It is understood that we always keep the identity, zero, and successor functions as initial functions, and that the substitution rule is unchanged. It will be shown that the functions given in the following table are sufficient to adjoin to the initial functions.

\begin{tabular}{|l|c|c|c|}
\hline & \multicolumn{2}{|c|}{ One Parameter } & No Parameter \\
\cline { 2 - 4 } & Recursion & Iteration & \\
\hline Mixed & - & - & $u+x, Q ;$ or $|u-x|$ \\
\hline Pure & $P$ & $Q$ & $u+x, E ;$ or $|u-x|, Q$ \\
\hline
\end{tabular}

In the table, the word mixed is used as the opposite of pure, and indicates that $B$ may depend on $x$. The dash in the cases of (mixed) recursion and (mixed) iteration indicates that no adjunctions are necessary. Two alternatives are given in the cases with no parameter. They are both valid even in the special case $a=0$. It is undersood that $u+x$ in the table indicates that we are to adjoin to the initial functions that function $F$ of two variables for which $F(u, x)=u+x$, and similarly for $|u-x|$.

Rózsa Péter has shown that recursion with one parameter is adequate. ${ }^{2}$ She has also shown that recursion with no parameter is sufficient to define all recursive functions, if three recursive functions $\left(u^{x}\right.$, the $x$ th prime $p_{x}$, and the exponent of the highest power of $p_{x}$ which divides $u$ ) are adjoined to the initial functions. ${ }^{8}$ We extend these results by showing that even iteration with one parameter is adequate, that simpler adjunctions can be made in the case of recursion with no parameter, and by discussing the various types of pure recursion.

Whether all the adjunctions made are actually necessary, I do not know. However, it is shown in $\S 8$ that in the case of recursion with no parameter, some function of more than one variable must be adjoined. In the case of pure recursion with no parameter, more ex-

${ }^{2} \mathrm{R}$. Péter Über den Zusammenhang der verschiedenen Begriffe der rekursiven Funktion, Math. Ann. vol. 110 (1934) pp. 612-632, especially pp. 619-620.

${ }^{3}$ R. Peter, Konstruktion nichtrekursiver Funktionen, Math. Ann. vol. 111 (1935) pp. 42-60, especially pp. 45-48. 
tensive results are obtained, which show in particular that $u+x$ and $Q$ would not be sufficient to adjoin.

In $\S 3$, we shall make the reduction to the various schemes with one parameter, and in $\$ 4$ to schemes with no parameter, by making certain adjunctions to the initial functions. The lemma of $\$ 5$ makes an essential reduction in the number of functions adjoined; but some of the more difficult definitions are reserved for $\$ 6$, where the proof of the results given in the above table is completed. In $\$ 7$, it is shown how all recursive functions of one variable may be defined without introducing functions of more than one variable. The necessity of the given adjunctions is discussed in $\$ 8$.

3. Reduction to schemes with one parameter. All of the reductions make use of the idea of associating an ordered pair of numbers with a single number. We must establish a one-to-one correspondence between all pairs of numbers and some numbers. This requires three functions $J(u, v), K x, L x$, satisfying the equations

$$
K J(u, v)=u, \quad L J(u, v)=v .
$$

If we have in addition

$$
J(K x, L x)=x,
$$

then a one-to-one correspondence is established between all pairs of numbers and all numbers. However, this condition is not needed, and we shall not assume it.

We shall assume that some suitable functions $J, K, L$ are adjoined to the initial functions. A possible set of functions is

$$
J(u, v)=(u+v)^{2}+u, \quad K x=E x, \quad L x=\left[x^{1 / 2}\right]-E x .
$$

These functions are all expressible in terms of

$$
u+x, \quad u-x, \quad x^{2}, \quad\left[x^{1 / 2}\right],
$$

where $u-x$ denotes any difference function. Thus it would be sufficient to adjoin these functions to the initial functions.

As a first reduction in the recursion scheme, we show that the number of parameters can be reduced to one. It is sufficient to show how to reduce the number of parameters by one, when there are two or more to begin with. In fact, it is sufficient to show how to reduce the number of parameters from two to one, since additional parameters could be carried through unchanged.

The given scheme has the form

$$
F(u, v, 0)=A(u, v), \quad F(u, v, S x)=B(u, v, x, F(u, v, x)) .
$$


It is seen that the function

$$
F^{\prime}(u, x)=F(K u, L u, x)
$$

is defined by the recursion scheme with one parameter

$$
F^{\prime}(u, 0)=A^{\prime} u, \quad F^{\prime}(u, S x)=B^{\prime}\left(u, x, F^{\prime}(u, x)\right),
$$

where

$$
A^{\prime} u=A(K u, L u), \quad B^{\prime}(u, x, y)=B(K u, L u, x, y) .
$$

Finally, we may put

$$
F(u, v, x)=F^{\prime}(J(u, v), x) .
$$

Thus we have shown that recursion with one parameter,

$$
F(u, 0)=A u, \quad F(u, S x)=B(u, x, F(u, x))
$$

is adequate. It remains to show how the $u$ and $x$ may be eliminated from $B(u, x, y)$. Let us first eliminate the $u$. (The opposite order could equally well be used.) We see that the function

$$
F^{\prime}(u, x)=J(u, F(u, x))
$$

is defined by the iteration with one parameter

$$
F^{\prime}(u, 0)=A^{\prime} u, \quad F^{\prime}(u, S x)=B^{\prime}\left(x, F^{\prime}(u, x)\right),
$$

where

$$
A^{\prime} u=J(u, A u), \quad B^{\prime}(x, y)=J(K y, B(K y, x, L y)) .
$$

Finally,

$$
F(u, x)=L F^{\prime}(u, x) .
$$

Thus iteration with one parameter,

$$
F(u, 0)=A u, \quad F(u, S x)=B(x, F(u, x))
$$

is also sufficient. Now the function

$$
F^{\prime}(u, x)=J(x, F(u, x))
$$

may be defined by

$$
F^{\prime}(u, 0)=A^{\prime} u, \quad F^{\prime}(u, S x)=B^{\prime} F^{\prime}(u, x),
$$

where

$$
A^{\prime} u=J(0, A u), \quad B^{\prime} y=J(S K y, B(K y, L y)) .
$$

Finally, 


$$
F(u, x)=L F^{\prime}(u, x) .
$$

Hence pure iteration with one parameter,

$$
F(u, 0)=A u, \quad F(u, S x)=B F(u, x),
$$

is sufficient to define all recursive functions. The further reduction of replacing $A u$ by $u$ was discussed in $\$ 2$.

4. Elimination of the last parameter. We shall now show how to eliminate the last parameter. For this purpose, we shall suppose that the pairing functions which we have adjoined to the initial functions have certain additional properties, namely:

$J(0,0)=0$, hence $K(0)=0$ and $L(0)=0$.

If $L S x \neq 0$, then $K S x=K x$ and $L S x=S L x$.

An example of suitable functions is

$$
J(u, v)=\left((u+v)^{2}+u\right)^{2}+v, \quad K x=E\left[x^{1 / 2}\right], \quad L x=E x,
$$

as is easily verified. These functions can be defined in terms of

$$
u+x, \quad u-x, \quad x^{2}, \quad\left[x^{1 / 2}\right],
$$

as was the case for the functions $J, K, L$ mentioned in $\$ 3$.

We now discuss the meaning of the conditions imposed. If we think of all pairs $(u, v)$ arranged in a table, where $u$ denotes the column number and $v$ denotes the row number, then the pair $(K x, L x)$ traverses the table in such a way that it starts at the top of column 0 and descends a certain number of steps; then it starts at the top of another column and descends a certain number of steps; and this process is repeated. Since we have pairing functions, every position in the table is traversed at some time. It is clear in fact that the equations $K x=u, L x=v$ have infinitely many solutions for $x$, one of which is called $J(u, v)$. A more precise description of the way the table is traced out for the particular $K$ and $L$ mentioned above could easily be given.

We showed in $\$ 3$ that iteration with one parameter is adequate to define all recursive functions. This may be taken in the form

$$
F(u, 0)=u, \quad F(u, S x)=B(x, F(u, x)) .
$$

The still more reduced scheme of pure iteration might be used, but there would be no advantage in this. We see that

can be defined by

$$
F^{\prime} x=F(K x, L x)
$$

$$
F^{\prime} 0=0, \quad F^{\prime} S x=B^{\prime}\left(x, F^{\prime} x\right),
$$


where

$$
B^{\prime}(x, y)= \begin{cases}K S x & \text { if } L S x=0, \\ B(L x, y) & \text { if } L S x \neq 0 .\end{cases}
$$

In obtaining this result, we have taken account of the special assumptions concerning the pairing functions made at the beginning of this section. If we had not imposed these conditions, we should not have an expression for $F^{\prime} S x$ in terms of $x$ and $F^{\prime} x$, but should have obtained a generalized type of recursion (with no parameter), which would require a further reduction. This less direct method was followed by R. Péter. We have finally

$$
F(u, x)=F^{\prime} J(u, x) .
$$

In this proof, we have obtained $B^{\prime}(x, y)$ by piecing together two known functions. The definition may be written explicitly as

$$
B^{\prime}(x, y)=0^{L S x} \cdot K S x+\operatorname{sgn} L S x \cdot B(L x, y) .
$$

This can be obtained by substitution if

$$
u+x, u \cdot 0^{x}
$$

are included in the initial functions.

We have thus shown that recursion with no parameter,

$$
F 0=0, \quad F S x=B(x, F x),
$$

is adequate to define all recursive functions, if suitable functions are adjoined to the initial functions.

The $x$ can be eliminated in the same way as in $\$ 3$. We see that

$$
F^{\prime} x=J(x, F x)
$$

can be defined by

$$
F^{\prime} 0=0, \quad F^{\prime} S x=B^{\prime} F^{\prime} x
$$

where

$$
B^{\prime} y=J(S K y, B(K y, L y))
$$

Finally,

$$
F x=L F^{\prime} x
$$

Thus we have reached the very simplest recursion scheme, pure recursion with no parameter, starting from 0 , that is,

$$
F 0=0, \quad F S x=B F x,
$$


which defines the function $F x=B^{x} 0$.

To make this reduction, it is sufficient to adjoin

$$
u+x, \quad u-x, \quad x^{2}, \quad\left[x^{1 / 2}\right], \quad u \cdot 0^{x}
$$

to the initial functions. Instead of $u \cdot 0^{x}$, we may of course adjoin the two functions $u x$ and $0^{x}$. Since

$$
u x=\left[\left\{(u+x)^{2}-u^{2}-x^{2}\right\} / 2\right],
$$

we may also say that the functions

$$
u+x, \quad u-x, \quad x^{2}, \quad 0^{x}, \quad[x / 2], \quad\left[x^{1 / 2}\right]
$$

are sufficient to adjoin.

\section{Sufficiency of adjoining certain functions.}

LEMMA. All recursive functions are definable by any of the recursion schemes considered, if the functions shown in the following table are adjoined to the initial functions.

\begin{tabular}{|l|c|c|c|}
\hline & \multicolumn{2}{|c|}{ One Parameter } & No Parameter \\
\cline { 2 - 3 } & Recursion & Iteration & \\
\hline Mixed & - & - & $u+x, u-x$ \\
\hline Pure & $P, Q$ & $P, Q$ & $u+x, u-x, Q$ \\
\hline
\end{tabular}

Proof. It will be sufficient to show that the functions listed at the end of $\$ 4$ can be defined in all cases.

$u+x$. This function is given in the cases with no parameter. Otherwise, we may define $u+x=S^{x} u$ by pure iteration.

$u-x$. A difference function is given in the cases with no parameter. Otherwise, $P$ is given in the pure cases, and may be defined by $P 0=0$, $P S x=x$ in the mixed cases; hence we may define the two difference functions

$$
u-x=P^{x} u, \quad|u-x|=(u-x)+(x-u) .
$$

$x^{2}$ (mixed cases). We may define $F x=x^{2}$ by

$$
F 0=0, \quad F S x=F x+2 x+1 .
$$

Here $2 x$ means $x+x$, and similarly below.

$x^{2}$ (pure cases). We first define the function 


$$
F x=x+2\left[x^{1 / 2}\right]
$$

Notice that

$$
F S x=S F x+2 Q S x .
$$

Now the following are equivalent: $S x$ is a square; $x$ is of the form $n^{2}+2 n ; F x$ is of the form $n^{2}+4 n ; F x+4$ is a square. Thus

$$
Q S x=Q(F x+4) \text {. }
$$

Hence we may put

$$
F x=B^{x} 0 \quad \text { with } B y=S y+2 Q(y+4) .
$$

Finally, we see that

$$
x^{2}=(S F)^{x} 0 .
$$

This definition makes use of $Q$ and $u+x$ but not of $P$.

$0^{x}$. We may define

$$
0^{x}=B^{x} 1 \quad \text { with } B y=0,
$$

except in the case with no parameter and with $a=0$. In this case, we first define

$$
\operatorname{sgn} x=B^{x} 0 \quad \text { with } B y=1,
$$

and then put $0^{x}=1-\operatorname{sgn} x$.

$[x / 2]$ (mixed cases). As in $\$ 1$, we first define

$$
D x=B^{x} 0 \quad \text { with } B y=0^{y},
$$

and then define $F x=[x / 2]$ by

$$
F 0=0, \quad F S x=F x+D x .
$$

$[x / 2]$ (pure cases). Here we first define

$$
T x=B^{x} 0 \quad \text { with } B y=0^{y}+2 \cdot 0^{(y-1)+(1-y)} .
$$

This is nearly the same definition given in $\$ 1$; we observe that the exponent $(y-1)+(1-y)$ vanishes if and only if $y=1$, no matter what difference function is used. Now if we define

$$
F x=B^{x} 0 \quad \text { with } B y=S y+T y,
$$

we see that

$$
[x / 2]=F x-x .
$$

$\left[x^{1 / 2}\right]$ (mixed cases). We define $F x=\left[x^{1 / 2}\right]$ as in $\$ 1$ by 


$$
F 0=0, \quad F S x=F x+0^{(S F x)^{2}-S x} .
$$

$\left[x^{1 / 2}\right]$ (pure cases). In defining $x^{2}$, we first defined

$$
F x=x+2\left[x^{1 / 2}\right] \text {. }
$$

We may put

$$
\left[x^{1 / 2}\right]=[(F x-x) / 2] .
$$

\section{Proof of the principal results.}

THEOREM. All recursive functions are definable by any of the recursion schemes considered, if the functions shown in the table in \$2 are adjoined to the initial functions.

Proof. This theorem may be reduced to the lemma of $\S 5$, by making the definitions (1)-(5) below. In carrying out the proof, definitions given in the proof of the lemma are referred to when they are applicable.

(1) Define $Q$ in terms of $P$ by pure recursion with one parameter. We may use the definitions of $u+x,|u-x|$, and $0^{x}$ from $\$ 5$. Now define $u x$ by the pure recursion

$$
u \cdot 0=0, \quad u \cdot S x=u x+u,
$$

and put $x^{2}=x x$. We next define a function $F(u, x)$ by the pure recursion

$$
F(u, 0)=0, \quad F(u, S x)=B(u, F(u, x)),
$$

where

$$
B(u, y)=S y+0\left|y^{2}-u\right| .
$$

That is, $F(u, S x)$ is obtained from $F(u, x)$ by adding 1 unless $\{F(u, x)\}^{2}=u$, in which case we add 2 . This can happen only if $x^{2}=u$. We thus see that

$$
F(u, x)= \begin{cases}x & \text { if } x^{2} \leqq u, \\ x+Q u & \text { if } x^{2}>u .\end{cases}
$$

Hence we may define

$$
Q u=F(u, S u)-S u \text {. }
$$

(2) Define $P$ in terms of $Q$ by pure iteration with one parameter. We may define $u+x$ and $x^{2}$ as in $\S 5$. Next define

$$
u \cdot 0^{x}=B^{x} u \quad \text { with } B y=0 ;
$$


in particular, $0^{x}=1 \cdot 0^{x}$. After defining $D x=B^{x} 0$ with $B y=0^{y}$, we can obtain the product $D x \cdot Q x$ from $u \cdot 0^{v}$ by putting $u=D x$ and $v=0^{Q x}$. Notice that $D x \cdot Q x$ is the characteristic function of odd squares. Let

$$
B y=S S y \cdot 0^{D y} \cdot Q y=\left\{\begin{array}{lr}
0 & \text { if } y \text { is an odd square. } \\
y+2 & \text { otherwise. }
\end{array}\right.
$$

Thus the function $B$ has the effect of increasing any number by 2 , except for an odd square, which is replaced by 0 . Now consider the function

$$
F x=B^{x}\left(x^{2}+x+1\right) .
$$

If $x$ is even and positive, then $x / 2$ additions of 2 are carried out to reach the next odd square $x^{2}+2 x+1$. The next application of $B$ gives 0 , and the remaining $(x / 2)-1$ steps produce $x-2$. Thus

$$
F x=x-2 \quad \text { if } x \text { is even and positive. }
$$

It is now easy to define $P x$. We have in fact

$$
P x=0^{0 x} \cdot 0^{D x} \cdot S F x+D x \cdot F S x .
$$

The first term can evidently be obtained by repeated substitution in $u \cdot 0^{v}$, and the second term can be obtained by substituting $u=F S x$ and $v=0^{D x}$. The correctness of the formula is easily checked by considering separately the cases $x=0, x$ even and positive, and $x$ odd.

(3) Define $u-x$ and $Q$ in terms of $u+x$ and $E$ by pure recursion with no parameter.

We first define sgn $x=B^{x} 0$ with $B y=1$, and then put

$$
0^{x}=E(2+2 \operatorname{sgn} x), \quad Q x=0^{E x} .
$$

We may then define $x^{2}$ as in $\$ 5$. Now

$$
E\left((u+x)^{2}+3 u+x+1\right)=u-x \quad \text { if } u \geqq x,
$$

since the preceding square is $(u+x)^{2}+2 u+2 x+1$. We have thus defined both $Q$ and a difference function.

(4) Define $u-x$ in terms of $u+x$ and $Q$ by recursion with no parameter.

Again define $\operatorname{sgn} x=B^{x} 0$ with $B y=1$, and then put

$$
0^{x}=Q S \operatorname{sgn} x, \quad D x=B^{x} 0 \text { with } B y=0^{y} .
$$

We may define $x^{2}$ as in $\$ 5$; the simpler definition for the mixed cases may now be used. Define $F x=\left[x^{1 / 2}\right]$ by

$$
F 0=0, \quad F S x=F x+Q S x,
$$


and $P x$ by $P 0=0, P S x=x$. Then define a new function $F x$ by

$$
F 0=0, \quad F S x=P\left(F x+2 D\left[x^{1 / 2}\right]\right) .
$$

We have

$$
F S x= \begin{cases}P F x & \text { if }\left[x^{1 / 2}\right] \text { is even } \\ S F x & \text { if }\left[x^{1 / 2}\right] \text { is odd }\end{cases}
$$

Hence

$$
F x=E x \quad \text { if }\left[x^{1 / 2}\right] \text { is odd, }
$$

so that

$$
F\left((2 u+2 x)^{2}+5 u+3 x+1\right)=u-x \quad \text { if } u \geqq x .
$$

(5) Define $u+x$ in terms of $|u-x|$ by pure recursion with no parameter. (Using this in place of (3) and (4) gives the second alternative of the table in $\$ 2$ for both types of recursion with no parameter.)

Since

$$
u+x=F(u, x)-\{(F(u, x)-u)-x\},
$$

for any function $F$ with $F(u, x) \geqq u+x$, we have only to define some such function. We first define

$$
\begin{gathered}
2 x=(S S)^{x} 0, \quad 2 x+1=S(2 x), \\
2^{x}-1=B^{x} 0 \text { with } B y=2 y+1, \quad 2^{x}=S\left(2^{x}-1\right) .
\end{gathered}
$$

We may then obtain the function

$$
F(u, x)=\left|2^{4 u}-2^{4 x+2}\right|
$$

by substitution. Now

$$
F(u, x)=\left|2^{2 u}-2^{2 x+1}\right| \cdot\left(2^{2 u}+2^{2 x+1}\right) .
$$

Since the first factor is not 0 , and the second factor is more than $u+x$, we have

$$
F(u, x)>u+x \text {. }
$$

7. Recursive functions of one variable. It is easy to see that all recursive functions of more than one variable can be obtained from recursive functions of one variable, the function $u+x$, and the various identity functions, by substitution alone. To see this, we shall make use of the pairing functions of $\$ 3$,

$$
J(u, v)=(u+v)^{2}+u, \quad K x=E x, \quad L x=\left[x^{1 / 2}\right]-E x .
$$


Suppose now that the recursive function $F(u, v)$ is given. If we let

$$
F^{\prime} x=F(K x, L x),
$$

then $F^{\prime} x$ is a recursive function of one variable. But

$$
F(u, v)=F^{\prime} J(u, v)=F^{\prime}\left((u+v)^{2}+u\right) .
$$

Thus $F(u, v)$ is obtained by substitution from the four functions

$$
F^{\prime} x, \quad x^{2}, \quad u+v, \quad I_{21}(u, v),
$$

of which the first two are recursive functions of one variable. Thus the proof is complete so far as functions of two variables are concerned. In the case of a function of more variables, a similar argument serves to reduce the number of variables by one, so that repeated use of the argument leads to the desired result.

The interest of this result is considerably enhanced by the fact that it is possible to define all recursive functions of one variable without making use of functions of more than one variable in the process. The third theorem below shows that this is the case.

THEOREM 1. The functions of one variable which are obtainable from certain initial functions by substitution and recursion of the form $F x=B^{x}$ are also obtainable if substitution is not allowed except for defining functions of one variable.

Proof. Let us call the functions thus obtainable from the given initial functions when substitution is used only for defining functions of one variable recursive, ${ }_{3}$, and functions obtainable by recursion and

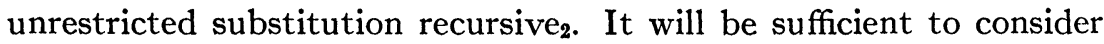
the class of functions obtainable by unrestricted substitution from

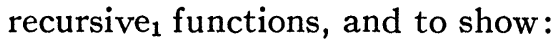

(1) All functions of one variable so obtained are recursive ${ }_{1}$.

(2) All recursive $e_{2}$ functions are obtained.

Proof of (1). In defining a function of one variable by successive substitutions, if the "innermost" substitutions are made first, only functions of one variable need be defined by substitution in the process. But a function of one variable defined by substitution from recursive $_{1}$ functions is recursive $_{1}$.

Proof of (2). Our family of functions includes all the initial functions and is closed with respect to substitution. Also, recursion of the form $F x=B^{x} a$ is allowed if $B$ is recursive, and hence by (1) for all functions $B$ of one variable in our family. Thus the family includes all recursive $e_{2}$ functions. 
THEOREM 2. If the initial functions include the identity and zero functions, and the function $u+x$, but no other functions of more than one variable, then all functions of one variable obtainable by substitution and recursion of the form $F x=B^{x} a$ are also obtainable by repeated use of the three formulas

$$
F x=A x+B x, \quad F x=B A x, \quad F x=B^{x} a
$$

to define $F$ when $A$ and $B$ are known functions of one variable.

Proof. By the preceding theorem, it is sufficient to use substitution to define only functions of one variable. Now substitution of functions of one variable into $I_{n k}$ gives one of the same functions; and such substitution into $O_{n}$ gives $O_{1}$, which is one of the initial functions. Hence only substitution into $u+x$ and into a function of one variable need be considered. In this way, we construct the functions $A x+B x$ and $B A x$.

Remark. Theorems 1 and 2 are also valid if we allow only recursion of the form $F x=B^{x} 0$.

THEOREM 3. All recursive functions of one variable can be obtained by starting with the two functions $S$ and $E$, and repeatedly using any of the formulas

$$
F x=A x+B x, \quad F x=B A x, \quad F x=B^{x} 0
$$

to construct a new function from known functions $A$ and $B$.

Proof. The identity and zero functions of one variable can be defined by

$$
I_{11} x=S^{x} 0, \quad O_{1} x=I_{11}^{x} 0 .
$$

Thus by Theorem 2, we can obtain all the functions of one variable which we could get by substitution and recursion $F x=B^{x} 0$ from all the identity and zero functions, $u+x, S$, and $E$. But by the theorem of $\$ 6$, all recursive functions can be so obtained.

Remarks. We could also prove a theorem similar to Theorem 2, with $|u-x|$ in place of $u+x$, and $|A x-B x|$ in place of $A x+B x$. Using this, a theorem analogous to Theorem 3 could be proved, with $Q$ in place of $E$, and $|A x-B x|$ in place of $A x+B x$.

8. Possibility of further improvements. Were all the adjunctions made in the Theorem of $\$ 6$ necessary? For example, in the case of pure recursion with one parameter, we adjoined $P$ to the initial functions. This adjunction is necessary if and only if $P$ itself cannot be defined. We are thus led to the interesting but apparently un- 
solved problem: Can predecessor be defined by substitution and pure recursion from the identity, zero, and successor functions? In a similar way, we may ask whether $Q$ can be defined by pure iteration.

In the case of recursion with no parameter, with or without $a=0$, some adjunction to the initial functions is necessary. Indeed, some function of more than one variable must be adjoined. For otherwise, each of the initial functions would depend on at most one of its variables, since the only functions of more than one variable which are given are the identity and zero functions. Now substitution applied to functions, each depending on at most one of its variables, can lead only to such a function; and our recursion scheme leads to a function of one variable. Thus no function depending on more than one variable can be defined; in particular, $u+x$ cannot be defined.

There remains the possibility that all recursive functions of one variable might be definable with no adjunctions. Finally, if it were possible to define $Q$ by adjoining only $u+x$, then the adjunction of $u+x$ would be sufficient for the definition of all recursive functions.

We come now to the simplest recursion scheme, pure recursion with no parameter, with or without $a=0$. We know that it is sufficient to adjoin $u+x$ and $E$, or $|u-x|$ and $Q$, to the initial functions. We shall show that it is not sufficient to adjoin $u+x$ and $Q$, and in particular that $P$ could not then be defined. This result is included in the more general theorem:

THEOREM. Suppose that to the identity, zero, and successor functions, we adjoin the function $u+x$, and any number of functions of one variable each of which has one or the other of the two properties:

$$
F x \geqq x, \quad F x \text { is bounded. }
$$

Then by repeated substitution and pure recursion with no parameter, no function of one variable not having one or the other of these two properties can be defined.

Proof. According to Theorem 2 of $\$ 7$, this reduces to the following lemma.

LemMa. From functions $A$ and $B$, each having one or the other of the two properties of the theorem, only functions having one of these properties can be defined by the three formulas

$$
F x=A x+B x, \quad F x=B A x, \quad F x=B^{x} a .
$$

Proof. (1) If $A x \geqq x$ or $B x \geqq x$, then $A x+B x \geqq x$. If both functions are bounded, then so also is their sum. 
(2) If either $A x$ or $B x$ is bounded, then so also is $B A x$. If both $A x \geqq x$ and $B x \geqq x$, then $B A x \geqq x$.

(3) If $B x$ is bounded, then so also is $B^{x} a$. Hence we may suppose $B x \geqq x$. If no two consecutive values of $B^{x} a$ are equal, then the function is strictly increasing, and hence $B^{x} a \geqq x$. If two consecutive values are equal, so also are all the following values, so that the function is bounded.

University of California AND

Princeton University 